\title{
Uso de plantas medicinales en las mujeres de la Sierra Centro, Ecuador durante el postparto
}

\author{
Use of medicinal plants in postpartum women in the Sierra Centro, Ecuador \\ Uso de plantas medicinais em puérperas na Sierra Centro, Equador
}

\author{
Silvia Inga Huilca \\ singa0170@uta.edu.ec \\ ORCID: 0000-0002-4193-6898
}

Universidad Técnica de Ambato, Ecuador

\author{
Alicia Zavala Calahorrano \\ am.zavala@uta.edu.ec \\ ORCID: 0000-0002-5670-7641
}

Universidad Técnica de Ambato, Ecuador

Recibido 16 de noviembre 2020 | Arbitrado y aceptado 24 de noviembre 2020 | Publicado 22 de diciembre 2020

\begin{abstract}
RESUMEN
Introducción: El empleo de plantas medicinales se encuentra extendido en diversas partes del mundo, dependiendo de la flora y fauna propia de cada región, específicamente la región altoandina en Ecuador las mujeres conservan la utilización de plantas con uso medicinal en la época del posparto. Objetivo: Conocer el uso de la medicina tradicional en mujeres durante la etapa de postparto. Método: Se realizó una investigación de tipo cualitativo, fenomenológico usando teoría fundamentada para el análisis de los datos, por medio de entrevistas semiestructuradas de las mujeres en postparto, familias y prestadores de salud tradicional. Se realizó entrevista a 15 mujeres en postparto y a 22 personas entre familiares y prestadores de salud del Cantón Pujilí- Cotopaxi - Ecuador. Resultados: Se realizó el análisis de los datos, encontrando que los principales usos de las plantas eran en infusión y en emplastos. Se dividió en 4 grupos de categorías 1) uso de plantas medicinales como infusión para purificación abdominal (purga), 2) uso de plantas medicinales como infusión para baño (baño postparto), 3) uso de plantas medicinales como infusión para limpieza de heridas (limpieza desinflamante), 4) uso de plantas medicinales como secretagogo (agüita para que no seque la leche). Conclusiones: El uso de plantas se encuentra es parte de la cultura y el desarrollo social y la comunidad encuentra cuatro categorías de uso de plantas medicinales en la época del postparto para las mujeres de la región altoandina. El personal de salud debe considerar el realizar más estudios en este campo específico contribuyendo así a preservar la salud de madre e hijo.
\end{abstract}

Palabras clave: Medicina tradicional; plantas medicinales, postparto, puerperio

\begin{abstract}
Introduction: The use of medicinal plants is widespread in various parts of the world, depending on the flora and fauna of each region, specifically the high Andean region in Ecuador, women preserve the use of plants with medicinal use in the postpartum period. Objective: To know the use of traditional medicine in women during the postpartum stage. Method: A qualitative, phenomenological research was carried out using grounded theory for data analysis, through semi-structured interviews of postpartum women, families and traditional health providers. An interview was conducted with 15 postpartum women and 22 people among family members and health providers from the Pujilí- Cotopaxi Canton - Ecuador. Results: The data analysis was carried out, finding that the main uses of the plants were in infusion and in plasters. It was divided into 4 groups of categories 1) use of medicinal plants as an infusion for abdominal purification (purge), 2) use of medicinal plants as an infusion for bath (postpartum bath), 3) use of medicinal plants as an infusion for wound cleaning (anti-inflammatory cleaning), 4) use of medicinal plants as a secretagogue (water so that the milk does not dry). Conclusions: The use of plants is part of culture and social development and the community finds four categories of use of medicinal plants in the postpartum period for women in the high Andean region. Health personnel should consider conducting further studies in this specific field, thus helping to preserve the health of mother and child.
\end{abstract}

Key words: Traditional medicine; medicinal plants; postpartum; puerperium
SI: Médico General graduada en la Escuela Superior Politécnica de Chimborazo. Desarrollo de proyectos comunitarios. Investigación en el área comunitaria Conferencista sobre uso de plantas medicinales. Cursando postgrado de Medicina Familiar y Comunitaria de la Universidad Técnica de Ambato. Universidad Técnica de Ambato, Ecuador.

AZ: Doctor en Medicina y Cirugía de la Universidad Central del Ecuador, Magister en Gerencia en Salud para el Desarrollo Local de la Universidad Técnica Particular de Loja Doctor of Phylosophy de Austrila de Loja, Doctor of Phylosophy de Australi Griffith University, Asesor de Despacho del Ministerio de Salud Pública, Especialista en Enfermedades Infecciosas con Potencial Epidémico y Pandémico 1 del Ministerio de Salud Pública, Ecuador. 
SI: Médico General graduada en la Escuela Superior Politécnica de Chimborazo. Desarrollo de proyectos comunitarios. Investigación en el área comunitaria Conferencista sobre uso de plantas Conferencista sobre uso de plantas medicinales. Cursando postgrado de Medicina Familiar y Comunitaria de la Universidad Técnica de Ambato. Universidad Técnica de Ambato, Ecuador.

AZ: Doctor en Medicina y Cirugía de la Universidad Central del Ecuador, Magister en Gerencia en Salud para el Desarrollo Local de la Universidad Técnica Particula de Loja, Doctor of Phylosophy de Australia Griffith University, Asesor de Despacho del Ministerio de Salud Pública, Especialista en Enform Enferme Epidémico y Pandémico 1 del Ministerio de Salud Pública, Ecuador.

\section{RESUMO}

Introdução: O uso de plantas medicinais é muito difundido em várias partes do mundo, dependendo da flora e da fauna de cada região, especificamente do alto andino do Equador, as mulheres preservam o uso de plantas com uso medicinal no período pós-parto. Objetivo: Conhecer o uso da medicina tradicional em mulheres no pós-parto. Método: Foi realizada uma pesquisa qualitativa fenomenológica, utilizando a teoria fundamentada para análise dos dados, por meio de entrevistas semiestruturadas com puérperas, familiares e profissionais de saúde tradicionais. Foi realizada uma entrevista com 15 puérperas e 22 pessoas entre familiares e profissionais de saúde do Cantão de Pujilí-Cotopaxi - Equador. Resultados: A análise dos dados foi realizada, constatando-se que os principais usos das plantas eram em infusão e em gesso. Foi dividido em 4 grupos de categorias 1) uso de plantas medicinais como infusão para purificação abdominal (purga), 2) uso de plantas medicinais como infusão para banho (banho pós-parto), 3) uso de plantas medicinais como infusão para limpeza de feridas (limpeza anti-inflamatória); 4) uso de plantas medicinais como secretagogo (água para que o leite não seque). Conclusões: $O$ uso de plantas faz parte da cultura e do desenvolvimento social e a comunidade encontra quatro categorias de uso de plantas medicinais no pós-parto para mulheres da região alta andina. Os profissionais de saúde devem considerar a realização de novos estudos nesta área específica, contribuindo assim para a preservação da saúde materno-infantil.

Palavras-chave: Medicina tradicional; plantas medicinais; pós-parto; puerpério

\section{INTRODUCCIÓN}

$\mathrm{L}$ a Organización Mundial de Salud determina que desde el año 2014 hasta el año 2023, la medicina tradicional se centrará en aprovechar los medicamentos complementarios y alternativos, en la atención médica $\mathrm{y}$ promover su uso seguro y eficaz. esto con el afán de satisfacer o complementar las necesidades médicas (1); por lo que acepta a nivel mundial el uso de plantas etnos medicinales como medicina alternativa durante el embarazo y la lactancia. Sin embargo, la identificación de plantas medicinales potencialmente útiles y la evaluación científica de su eficacia y seguridad al momento no se encuentran bien identificadas (2).

Alrededor de 30.000 especies de plantas se utilizan con fines curativos en diversas culturas del mundo, las hierbas medicinales, desde tiempos inmemoriales, prácticamente en todas las culturas como fuente de medicina (3). El uso de plantas medicinales con fines curativos es tan antiguo como la humanidad misma. El uso generalizado de remedios a base de hierbas para el cuidado de la salud se ha descrito en textos antiguos como los Vedas y la Biblia (4). Estos libros contienen las experiencias de miles de años de ensayo y error en el descubrimiento de propiedades curativas. Actualmente, con el desarrollo de la ciencia están en segundo plano; sin embargo, en muchos países sigue siendo la opción económica para acceder a la salud.

El periodo de la maternidad, y durante el puerperio, no hay evidencia clara que permita un manejo de salud integral con pertenencia étnica. . Durante esta etapa existe incertidumbre de la nueva madre frente a la sociedad y la integración de un nuevo miembro del hogar. La falta de compresión de un sistema de salud, la influencia familiar y los escasos recursos económicos son los principales factores que muchas de las mujeres inciden en el uso de medicina herbaria tradicional. Sin embargo, la advertencia de muchos fármacos contraindicados para embarazo y 
lactancia fomentan el uso de plantas medicinales de forma segura y natural dentro del periodo de postparto (5).

Los principales síntomas y signos que padecen las mujeres durante el postparto es el sangrado vaginal por la expulsión de loquios, insomnio, ansiedad, depresión, incontinencia y retención urinaria, síndrome de leche insuficiente (6), inmunosupresión, debilidad muscular y dolor (7). Los mismos que se encuentran asociados a cambios biológicos tanto físicos como mentales. En donde se produce una involución de los cambios que se produce para sustentar el embrazo acompañados de la inclusión social y las responsabilidades provocan una crisis en la mujer durante esta etapa.

El Ecuador, al ser un país biodiverso en flora y cultura acompañado de ser un país en vías de desarrollo con un sistema de salud todavía no bien sustentado (8). Lo cual permite que en la actualidad el uso de plantas medicinales sea común. La pluriculturalidad habilita que se desarrollen algunas prácticas y uso de plantas en diferentes regiones. Un ejemplo es la región sierra, con 13 pueblos aborígenes (9). La sierra centro cuenta con aproximadamente 6 pueblos cuyas costumbres son muy similares debido a la cercanía que se encuentran. Durante el postparto estos pueblos practican la "Dieta" que se trata del descanso de la mujer en el domicilio. Durante esta etapa el uso de plantas medicinales tendrá diferentes objetivos que se detallará en el presente trabajo.

Ecuador continental se encuentra dividido en tres regiones costa, sierra y oriente. El estudio se realizó en la provincia de Cotopaxi cantón Pujilí que corresponde a la sierra centro. Esta área geográfica se caracteriza por la prevalencia de población que mantienen identidad y costumbres culturales.

El estudio tiene como objetivo determinar el uso de plantas medicinales y analizar las experiencias poblacionales en uso de plantas con fines medicinales, desde el contexto cultural y comunitario. Logrando con ello, destacar la importancia de esta práctica en la región de la sierra ecuatoriana y zonas rurales donde los ancestral cobra vida y se mantiene a pesar del tiempo.

\section{MATERIALES Y MÉTODOS}

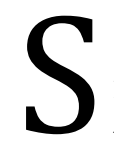

e trató de un estudio cualitativo, fenomenológico, descriptivo, transversal de campo para conocer los diferentes usos de plantas medicinales durante el postparto. Como técnicas de recolección y el muestreo de datos se utilizó observación indirecta mediante el acompañamiento a los equipos de salud en el seguimiento de mujeres postparto. Con el que se logró seleccionar 15 mujeres que cursaban su postparto con la utilización de plantas medicinales, al cual se le denominó grupo directo. Se aplicó muestreo en bola de nieve al grupo directo para identificar al grupo indirecto. El grupo indirecto se conformó por 22 personas entre hombres $\mathrm{y}$ mujeres que influyeron en el conocimiento sobre la utilización de plantas medicinales al grupo directo. A los dos grupos se aplicó la técnica de entrevista semiestructurada para la recolección de datos. Respetando los derechos de confidencialidad y libertad de participación. Además, las entrevistas fueron trascritos a medios físicos $\mathrm{y}$ digitales. Se analizaron manualmente dando interpretación a las expresiones de cada participante. Los datos se examinaron mediante métodos cualitativos en la 
construcción del fenómeno mediante teoría fundamentada.

\section{RESULTADOS}

$\mathrm{E}$ l periodo postparto desde el punto de vista cultural se denominado DIETA. La DIETA es un periodo de un mes, en el que las mujeres cumplieron un reposo en el domicilio. El reposo se lo realizó para recuperar la energía perdida durante el proceso de parto. La energía es representada por el calor como sensación térmica de bienestar. El objetivo de la recuperación fue ejecutar acciones para promover el calor y recuperar a un cuerpo debilitado. Entre las actividades que crean mayor cantidad de calor son el uso de plantas medicinales en infusiones y baños.

...después del parto, las hierbas sirven para endurecer el cuerpo porque queda débil por perder muchas sangre y eso se les ayuda con las hierbas tanto para tomar tanto para bañar. Persona del grupo indirecto que asesoro el uso de plantas a la mujer postparto 6 .

La familia fue la principal promotora del uso de plantas medicinales. Las madres y los esposos de las mujeres postparto acudieron a los agentes tradicionales de la salud para instruirse en las preparaciones. Los agentes tradicionales de la salud son parteras, hierbateros o yuyeros y sabios comunitarios. Quienes son parte de la comunidad, brindaron un servicio de medicina ancestral desde el conocimiento vivencial. Las parteras acudían al domicilio a preparar las infusiones y dar de tomar a la parturienta. Los hierbateros o yuyeros vendieron el conjunto de hierbas para preparar las infusiones. Los sabios instruyen la recolección silvestre de las diversas especies de plantas para las preparaciones.

...al salir del hospital venimos a
la casa de mi mamá, con mi
esposo me cuidaron con agüitas
de campo que aprendió mi
mamá y compro en el mercado
con los yuyeros. Mujer
postparto 13 .

Los hierbateros y los sabios comunitarios dividían las propiedades de las plantas en frías, calientes y frescas. Las plantas frías son las que por sus propiedades van a generar frío en el cuerpo. Las plantas cálidas van a producir en el cuerpo aumento de la temperatura. Las plantas frescas son las que no desencadenan ni frío, ni calor tienen un carácter refrescante para vitalizar $\mathrm{y}$ mineralizar el cuerpo.

Basados en estos principios, se prepararon las infusiones con ciertas especies de plantas. Las infusiones abarcaron diferentes finalidades destinadas a la recuperación y evitar complicaciones durante el puerperio. Para esquematizar la forma de empleo se la divide de la siguiente manera:

- Uso de plantas Medicinales como infusión para purificación Abdominal (purga).

- Uso de plantas Medicinales como infusión para baño (baño postparto).

- Uso de plantas Medicinales como infusión para limpieza de heridas (limpieza desinflamante).

- Uso de plantas Medicinales como secretagogo (agüita para que no seque la leche). 
Uso de plantas medicinales como infusión para purificación abdominal (purga)

La totalidad de mujeres entrevistadas independientemente del tipo de parto, consumieron un preparado en infusión denominado PURGA. La purga fue una bebida empleada para fomentar la eliminación de loquios hemáticos $\mathrm{y}$ residuos placentarios después del parto. Las puérperas ingirieron esta bebida durante 9 días en la mañana. El consumo de esta bebida según las recomendaciones de los sabios debe ser rápido, para mantener el calor de su preparación.

...el agua de purgas es para botar toda esa sangre que esta mala adentro de uno, así hacían tomar calientito a soplos por 9 días después del parto. Mujer postparto 2 . familias acudieron al sabio para recibir orientación en la recolección de plantas. La segunda vía, las familias acudieron a los hierbateros para comprar y orientarse en la preparación de la bebida. En la preparación se procedió a dividir la parte de la planta a ser empleada. Colocaron agua para ebullición en una olla grande. Las raíces y los troncos por su consistencia dura son triturados y colocados en primer lugar en el agua. Posterior colocaron las hojas y ramas cuando haya realizado una ebullición. Finalmente, se colocaron las flores y se procede a retirar del fuego. Las especies de plantas utilizadas en la preparación son muy diversas y depende de la costumbre de la familia y la experiencia de los sabios. La totalidad de plantas registradas en las entrevistas para la preparación de la purga se detalla en la Tabla 1.

La recolección de las plantas fue realizada por dos vías. La primera vía, las

Tabla 1. Plantas Medicinales utilizadas para la Infusión

\begin{tabular}{lllllll}
\hline & & & & & \\
& & & &
\end{tabular}




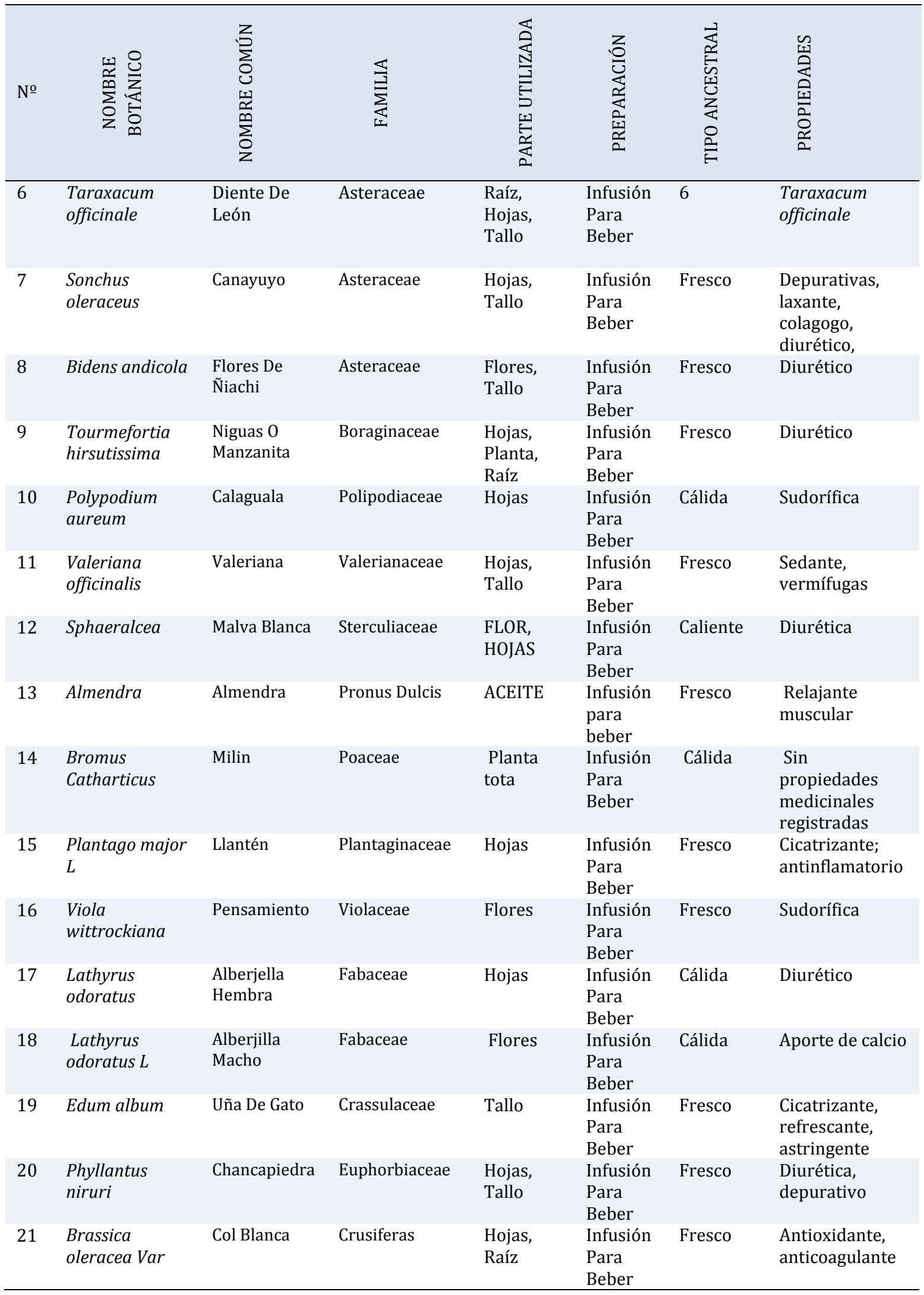




\begin{tabular}{|c|c|c|c|c|c|c|c|}
\hline № & 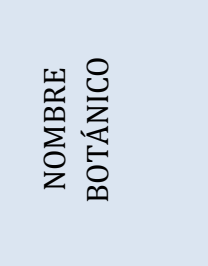 & 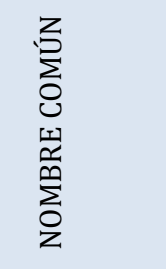 & 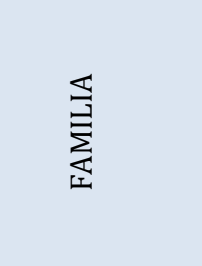 & 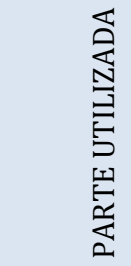 & 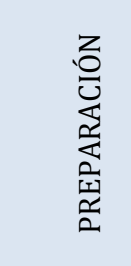 & 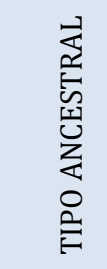 & 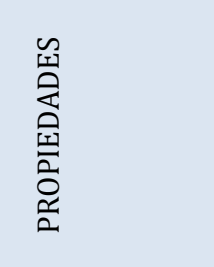 \\
\hline 22 & $\begin{array}{l}\text { Peperomia } \\
\text { peltigera }\end{array}$ & $\begin{array}{l}\text { Pata Con } \\
\text { Yuyo }\end{array}$ & Piperaceae & Hojas & $\begin{array}{l}\text { Infusión } \\
\text { Para } \\
\text { Beber }\end{array}$ & Fresco & Depurativo \\
\hline 23 & $\begin{array}{l}\text { Aerva } \\
\text { sanguinolenta }\end{array}$ & Escancel & $\begin{array}{l}\text { Amaranthace } \\
\text { ae }\end{array}$ & $\begin{array}{l}\text { Hojas, } \\
\text { Raíces, } \\
\text { Flores }\end{array}$ & $\begin{array}{l}\text { Infusión } \\
\text { Para } \\
\text { Beber }\end{array}$ & Fresco & Diuréticas \\
\hline 24 & $\begin{array}{l}\text { Borago } \\
\text { officinalis }\end{array}$ & Borraja & Boraginaceae & Hojas & $\begin{array}{l}\text { Infusión } \\
\text { Para } \\
\text { Beber }\end{array}$ & Fresco & $\begin{array}{l}\text { Sudorífica, } \\
\text { depurativa, } \\
\text { descongestiva, } \\
\text { refrescante }\end{array}$ \\
\hline 25 & $\begin{array}{l}\text { Equisetum } \\
\text { arvense }\end{array}$ & $\begin{array}{l}\text { Cola De } \\
\text { Caballo }\end{array}$ & Equistaceae & $\begin{array}{l}\text { Tallo, } \\
\text { Hoja }\end{array}$ & $\begin{array}{l}\text { Infusión } \\
\text { Para } \\
\text { Beber }\end{array}$ & Fresco & $\begin{array}{l}\text { Regenerarte, } \\
\text { cicatrizante, } \\
\text { diurética }\end{array}$ \\
\hline 26 & $\begin{array}{l}\text { Arracacia } \\
\text { xanthorrhiza }\end{array}$ & Zanahoria & Apiaceae & Hoja & $\begin{array}{l}\text { Infusión } \\
\text { Para } \\
\text { Beber }\end{array}$ & Fresco & $\begin{array}{l}\text { Diurético, } \\
\text { estimulante, }\end{array}$ \\
\hline 27 & Leptochloa & Paja Blanca & Poaceae & $\begin{array}{l}\text { Hojas Y } \\
\text { Raíz }\end{array}$ & $\begin{array}{l}\text { Infusión } \\
\text { Para } \\
\text { Beber }\end{array}$ & Fresco & \\
\hline 28 & $\begin{array}{l}\text { Luma } \\
\text { apiculata }\end{array}$ & Arrayan & Myrtaceae & $\begin{array}{l}\text { Hojas, } \\
\text { Ramas }\end{array}$ & $\begin{array}{l}\text { Infusión } \\
\text { Para } \\
\text { Beber }\end{array}$ & Fresco & $\begin{array}{l}\text { Astringentes, } \\
\text { antisépticos }\end{array}$ \\
\hline 29 & Zea Maíz & Choclo & Poaceae & $\begin{array}{l}\text { Pelo Del } \\
\text { Choclo }\end{array}$ & $\begin{array}{l}\text { Infusión } \\
\text { Para } \\
\text { Beber }\end{array}$ & Fresco & Depurativo \\
\hline 30 & $\begin{array}{l}\text { Taraxacum } \\
\text { officinale }\end{array}$ & Taraxaco & Asteraceae & Hojas & $\begin{array}{l}\text { Infusión } \\
\text { Para } \\
\text { Beber }\end{array}$ & Fresco & Diuréticas \\
\hline 31 & $\begin{array}{l}\text { Caléndula } \\
\text { officinalis }\end{array}$ & Caléndula & Asteraceae & $\begin{array}{l}\text { Hojas, } \\
\text { Flores }\end{array}$ & $\begin{array}{l}\text { Infusión } \\
\text { Para } \\
\text { Beber }\end{array}$ & Fresco & $\begin{array}{l}\text { Cicatrizante, } \\
\text { regenerador de } \\
\text { células }\end{array}$ \\
\hline 32 & $\begin{array}{l}\text { Origanum } \\
\text { vulgare }\end{array}$ & Orégano & Lamiaceae & $\begin{array}{l}\text { Hojas, } \\
\text { Tallo }\end{array}$ & $\begin{array}{l}\text { Infusión } \\
\text { Para } \\
\text { Beber }\end{array}$ & Fresco & $\begin{array}{l}\text { Antioxidante, } \\
\text { antitumoral, } \\
\text { antiséptica }\end{array}$ \\
\hline
\end{tabular}

Fuente: nombre común, parte utilizada, preparación y tipo ancestral extraído de las entrevistas realizada. Nombre Botánico, Familia y Propiedades de la base de datos botánicos de la siguiente página http://www.conabio.gob.mx/malezasdemexico/2inicio/home-malezas-mexico.htm. 
...las aguas de purgas constan de 22 plantas que se prepara en una olla grande colocando el atado que se les vende y se da a tomar a soplos. Persona del grupo indirecto que asesoró el uso de plantas a la mujer postparto 8 .

\section{Uso de plantas medicinales como infusión para baño (baño postparto)}

Las familias impidieron que las mujeres postpartos se bañen con agua simple para evitar dolores articulares futuros. El baño lo realizaron después de una semana de reposo en el domicilio con una infusión de plantas. Con el objetivo de evitar que el cuerpo disminuya su temperatura y enferme.

..."se debe hacer bañar en la
dieta con las plantas nativas
como dicen los mayores para
que nos endure el cuerpo para
que no quede con dolor de
huesos" Persona del grupo
indirecto que asesoró el uso de
plantas a la mujer postparto 5.

El baño se lo preparaba cocinando una serie de especies de plantas en ollas grandes. En cantidades que abastezcan para ducharse totalmente. Las plantas utilizadas de igual forma son recolectadas por los dos métodos descritos en la purga. Se colocó las especies de plantas a cocinar, todas a la vez hasta que lleguen al punto de ebullición. El preparado se coloca en tinas grandes para que enfríe y se proceda a bañar. No está permitido el uso de jabones y esencias durante el proceso. La limpieza fue realizada frotando el cuerpo con los residuos de plantas producto de la cocción.

$$
\begin{aligned}
& \text {..."en una olla grande ya } \\
& \text { cuando está hirviendo se ponen } \\
& \text { todas las hierbitas, una vez que } \\
& \text { ya esté listo le pasa a un tacho } \\
& \text { grande y se le deja que se } \\
& \text { tiemple no se mezcla con agua } \\
& \text { fría solo que se enfrié con el } \\
& \text { ambiente". Persona del grupo } \\
& \text { indirecto que asesoró el uso de } \\
& \text { plantas a la mujer postparto } 2 .
\end{aligned}
$$

El baño tuvo un significado de purificación y renovación de energía positiva. Se lo realizó cada semana hasta completar 1 mes de cumplimiento de la dieta. Se lo complemento con una alimentación específica en cada baño. Las diversas especies empleadas en la preparación del baño se detallan en la Tabla 2. 
Tabla 2. Plantas Medicinales utilizadas en el Baño

\begin{tabular}{|c|c|c|c|c|c|c|c|}
\hline № & 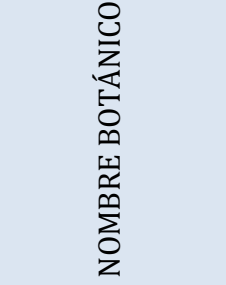 & 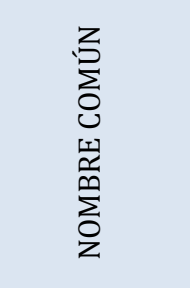 & 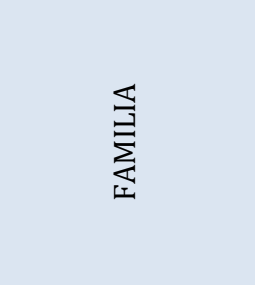 & 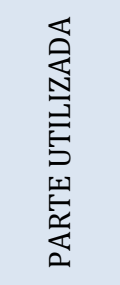 & 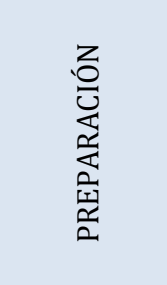 & 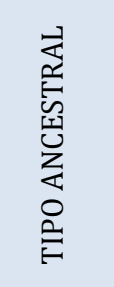 & 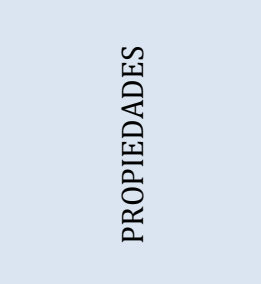 \\
\hline 1 & Laurus novilis & Laurel & Lauraceae & $\begin{array}{l}\text { Hojas y } \\
\text { Bayas }\end{array}$ & $\begin{array}{l}\text { Infusión } \\
\text { Para Baño }\end{array}$ & Cálida & $\begin{array}{l}\text { Digestivo, } \\
\text { antiséptico, } \\
\text { diurético }\end{array}$ \\
\hline 2 & $\begin{array}{l}\text { Oreopanax } \\
\text { ecuadorensi }\end{array}$ & Pumamaqui & Araliaceae & $\begin{array}{l}\text { Hojas y } \\
\text { Ramas }\end{array}$ & $\begin{array}{l}\text { Infusión } \\
\text { Para Baño }\end{array}$ & Cálida & Terapéuticas \\
\hline 3 & Juglans & Nogal & Juglandaceae & Hojas & $\begin{array}{l}\text { Infusión } \\
\text { Para Baño }\end{array}$ & Cálida & $\begin{array}{l}\text { Antiséptica, } \\
\text { diaforética, } \\
\text { antidepresiva }\end{array}$ \\
\hline 4 & $\begin{array}{l}\text { Salvia } \\
\text { rosmarinus }\end{array}$ & Romero & Lamiaceae & $\begin{array}{l}\text { Hojas y } \\
\text { Ramas }\end{array}$ & $\begin{array}{l}\text { Infusión } \\
\text { Para Baño }\end{array}$ & Cálida & $\begin{array}{l}\text { Astringente, } \\
\text { colagoga, } \\
\text { diurética }\end{array}$ \\
\hline 5 & $\begin{array}{l}\text { Buddleja } \\
\text { globosa }\end{array}$ & Matico & Scrophulariaceae & Hojas & $\begin{array}{l}\text { Infusión } \\
\text { Para Baño }\end{array}$ & Fresco & $\begin{array}{l}\text { Astringente, } \\
\text { cicatrizante }\end{array}$ \\
\hline 7 & $\begin{array}{l}\text { Chamaemelum } \\
\text { nobile }\end{array}$ & Manzanilla & Asteraceae & Flores & $\begin{array}{l}\text { Infusión } \\
\text { Para Baño }\end{array}$ & Cálida & $\begin{array}{l}\text { Antiinflamatorias, } \\
\text { antiespasmódicas, } \\
\text { emenagogas }\end{array}$ \\
\hline 8 & $\begin{array}{l}\text { Eucaliptus } \\
\text { citriodora } \\
\text { Hook }\end{array}$ & $\begin{array}{l}\text { Eucalipto } \\
\text { Aromático }\end{array}$ & Myrtaceae & $\begin{array}{l}\text { Hojas y } \\
\text { Ramas }\end{array}$ & $\begin{array}{l}\text { Infusión } \\
\text { Para Baño }\end{array}$ & Cálida & $\begin{array}{l}\text { Expectorante, } \\
\text { antiséptica Y } \\
\text { descongestionante }\end{array}$ \\
\hline 9 & $\begin{array}{l}\text { Myrtus } \\
\text { communis L }\end{array}$ & Arrayan & Myrtalece & Hojas & $\begin{array}{l}\text { Infusión } \\
\text { Para Baño }\end{array}$ & Cálida & $\begin{array}{l}\text { Astringentes, } \\
\text { antisépticas, } \\
\text { hemostáticas }\end{array}$ \\
\hline 10 & Leptochloa & Paja Blanca & Poaceae & Hojas & $\begin{array}{l}\text { Infusión } \\
\text { Para Baño }\end{array}$ & Fresco & \\
\hline 11 & Rosa Spp & $\begin{array}{l}\text { Rosas } \\
\text { (Flores) }\end{array}$ & Rosáceas & Hojas & $\begin{array}{l}\text { Infusión } \\
\text { Para Baño }\end{array}$ & Fresco & $\begin{array}{l}\text { Cicatrizante, } \\
\text { astringente, } \\
\text { antiséptica }\end{array}$ \\
\hline 12 & Ficus carica & Higo & Moráceas & Hojas & $\begin{array}{l}\text { Infusión } \\
\text { Para Baño }\end{array}$ & Cálida & $\begin{array}{l}\text { Digestivo, } \\
\text { diurético }\end{array}$ \\
\hline 14 & Urtica diodica & Ortiga & Urticaceae & $\begin{array}{l}\text { Hojas y } \\
\text { Raíces }\end{array}$ & $\begin{array}{l}\text { Infusión } \\
\text { Para Baño }\end{array}$ & Cálida & $\begin{array}{l}\text { Diurética, } \\
\text { depurativa, } \\
\text { remineralizarte }\end{array}$ \\
\hline 15 & $\begin{array}{l}\text { Brassica } \\
\text { rapal.Var.Rapa }\end{array}$ & Nabo & Brassicaceae & $\begin{array}{l}\text { Hojas, } \\
\text { Flores }\end{array}$ & $\begin{array}{l}\text { Infusión } \\
\text { Para Baño }\end{array}$ & Fresco & Diurético \\
\hline
\end{tabular}

Fuente: nombre común, parte utilizada, preparación y tipo ancestral extraído de las entrevistas realizada. Nombre Botánico, Familia y Propiedades de la base de datos botánicos de la siguiente página http://www.conabio.gob.mx/malezasdemexico/2inicio/home-malezas-mexico.htm. 


\section{Uso de plantas medicinales como infusión para limpieza de heridas (limpieza desinflamante)}

Aseo de mamas: El aseo en un parto sin complicaciones para la madre o el niño se lo realizó a partir de los 8 días. Sin embargo, algunas mujeres no consideran necesario el aseo de estos. El aseo de mamas solo se realizó cuando se presentó complicaciones en la madre o el niño. En el caso de las madres se lo realizó cuando hay mastitis y eversión de pezón. En el caso de los niños, se lo realizó cuando egresan de neonatología o presentan Muguet. En estos casos las mujeres utilizan la infusión de manzanilla como desinflamante de mamas.

\section{..."con agua desinflamantes de manzanilla me limpiaba los senos cada que iba a dar de lactar 3 veces al día porque se me agrietaban" Mujer postparto 1.}

Aseo de genitales: la ejecución del aseo depende del tipo de parto que se genere. En las mujeres cuyo parto fue domiciliario, la partera acudía al domicilio en días posteriores para realizar la limpieza. En el parto institucional las mujeres fueron aseadas en las unidades. Cuando llegaron a casa este aseo fue impuesto su ejecución con preparado especial. El preparado de plantas se lo realiza en infusión con principios desinflamatorios. El lavado lo continúa realizando cada dos días hasta las tres semanas o el momento del baño. También se utiliza otros productos adicionales a las infusiones para ayudar a la cicatrización. Estos productos son: mertiolate, agua bendita y el agua residual del baño del neonato.

...me lavaba y me botaba agua desinflamante, me fregaba con agüita de manzanilla dos veces al día. Mujer postparto 15.

Limpieza de herida quirúrgica: Para las mujeres fue difícil aceptar poseer una herida. La herida representa una debilidad de cuerpo que hay que cuidar. La cesárea y la episiotomía implicaron la utilización de fomentos e infusiones que permitan la cicatrización. Principalmente la herida de cesárea requiere limpieza con aguas desinflamantes.

El listado de infusiones y emplastos utilizados para la limpieza de mamas, genitales y heridas quirúrgicas se detalla en la Tabla 3. 
Tabla 3. Plantas Medicinales para el Aseo Diario

\begin{tabular}{|c|c|c|c|c|c|c|c|}
\hline № & 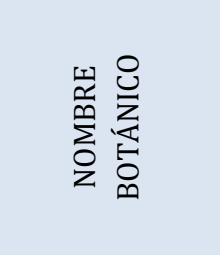 & 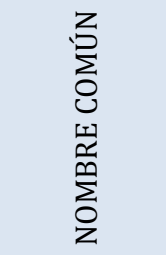 & 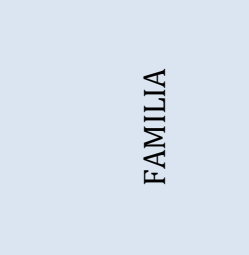 & 贸志 & 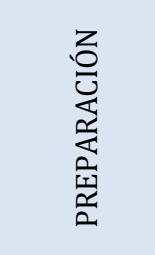 & 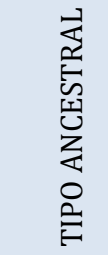 & 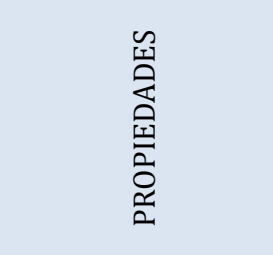 \\
\hline 1 & $\begin{array}{l}\text { Clematis } \\
\text { dioica }\end{array}$ & $\begin{array}{l}\text { Hierva de } \\
\text { Ángel }\end{array}$ & Cuscutaceae & $\begin{array}{l}\text { Toda la } \\
\text { Planta }\end{array}$ & $\begin{array}{l}\text { Infusión } \\
\text { para baño }\end{array}$ & Fresco & $\begin{array}{l}\text { Depurativo, } \\
\text { diurético, hepático }\end{array}$ \\
\hline 2 & $\begin{array}{l}\text { Chamaemelum } \\
\text { nobile }\end{array}$ & Manzanilla & Asteraceae & $\begin{array}{l}\text { Hojas, } \\
\text { Tallo }\end{array}$ & $\begin{array}{l}\text { Infusión } \\
\text { para baño }\end{array}$ & Fresco & $\begin{array}{l}\text { Antiinflamatorias, } \\
\text { antiespasmódicas, } \\
\text { emenagogas }\end{array}$ \\
\hline 3 & $\begin{array}{l}\text { Buddleja } \\
\text { globosa }\end{array}$ & Matico & Scrophulariaceae & Hojas & $\begin{array}{l}\text { Infusión } \\
\text { para baño }\end{array}$ & Fresco & $\begin{array}{l}\text { Astringente, } \\
\text { cicatrizante }\end{array}$ \\
\hline 4 & $\begin{array}{l}\text { Melissa } \\
\text { officinalis }\end{array}$ & Toronjil & Lamiaceae & Hojas & $\begin{array}{l}\text { Infusión } \\
\text { para baño }\end{array}$ & Fresco & Antiespasmódica \\
\hline 5 & $\begin{array}{l}\text { Malva } \\
\text { sylvestris }\end{array}$ & Malva & Malvaceae & Hojas & $\begin{array}{l}\text { Infusión } \\
\text { para baño }\end{array}$ & Fresco & $\begin{array}{l}\text { Cicatrizante, } \\
\text { calmante, digestiva }\end{array}$ \\
\hline 7 & $\begin{array}{l}\text { Plantago } \\
\text { major L }\end{array}$ & Llantén & Plantaginaceae & $\begin{array}{l}\text { Toda la } \\
\text { Planta }\end{array}$ & $\begin{array}{l}\text { Infusión } \\
\text { para baño }\end{array}$ & Fresco & $\begin{array}{l}\text { Cicatrizante; } \\
\text { Antinflamatorio }\end{array}$ \\
\hline 8 & $\begin{array}{l}\text { Urtica } \\
\text { diodica }\end{array}$ & Ortiga & Urticaceae & $\begin{array}{l}\text { Hojas, } \\
\text { Tallo }\end{array}$ & $\begin{array}{l}\text { Infusión } \\
\text { para baño }\end{array}$ & Fresco & $\begin{array}{l}\text { Diurética, } \\
\text { depurativa, } \\
\text { remineralízante }\end{array}$ \\
\hline 9 & $\begin{array}{l}\text { Linum } \\
\text { usitatissimum }\end{array}$ & Linaza & Lino & Semilla & $\begin{array}{l}\text { Infusión } \\
\text { para baño }\end{array}$ & Fresco & Laxante \\
\hline
\end{tabular}

Fuente: nombre común, parte utilizada, preparación y tipo ancestral extraído de las entrevistas realizada. Nombre Botánico, Familia y Propiedades de la base de datos botánicos de la siguiente página http://www.conabio.gob.mx/malezasdemexico/2inicio/home-malezas-mexico.htm.

\section{Uso de plantas medicinales como segretagogo (agüita para que no seque la leche)}

Según la sabiduría popular existen plantas medicinales para incrementar la producción láctea, la planta más utilizada es el Paraguay. Las mujeres recomendaban y utilizaban la infusión de Paraguay como tratamiento de la hipogalactia. La infusión de debe beber los primeros 15 días en la mañana y en la noche, el objetivo es extraer el frío del cuerpo ocasionado por el parto y los resfriados mal curados. La caracterización de las plantas que incrementan la producción de leche materna se puede ver en la Tabla 4. 
Tabla 4. Plantas Medicinales para Mejorar la Lactancia Materna

\begin{tabular}{|c|c|c|c|c|c|c|c|}
\hline № & 留 & 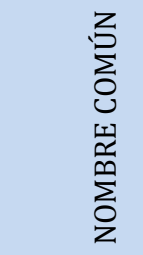 & $\underset{\sum}{\stackrel{\Xi}{\Xi}}$ & 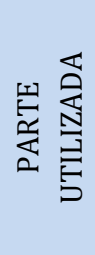 & 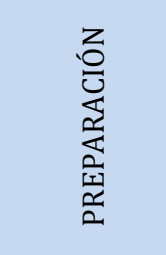 & 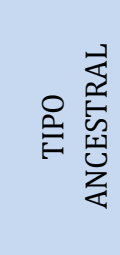 & 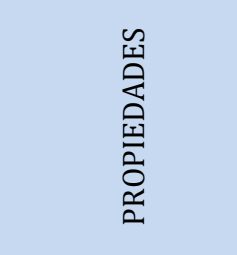 \\
\hline 1 & $\begin{array}{l}\text { Brunfelsia } \\
\text { pauciflora }\end{array}$ & Paraguay & Solnaceae & Hojas & $\begin{array}{l}\text { Infusión } \\
\text { Para Beber }\end{array}$ & Cálida & $\begin{array}{l}\text { Broncodilatador, } \\
\text { diurético }\end{array}$ \\
\hline 2 & Juglans regia & Nogal & Juglandaceae & Hojas & $\begin{array}{l}\text { Infusión } \\
\text { Para Beber }\end{array}$ & Cálida & $\begin{array}{l}\text { Relajante, } \\
\text { cicatrizante, } \\
\text { hipoglucémicas }\end{array}$ \\
\hline 3 & $\begin{array}{l}\text { Euphorbia } \\
\text { lactiflua }\end{array}$ & Lecheron & Euforbiaceas & Hojas & $\begin{array}{l}\text { Infusión } \\
\text { Para Beber }\end{array}$ & Cálida & $\begin{array}{l}\text { Eméticas, } \\
\text { catárticas }\end{array}$ \\
\hline
\end{tabular}

Fuente: nombre común, parte utilizada, preparación y tipo ancestral extraído de las entrevistas realizada. Nombre Botánico, Familia y Propiedades de la base de datos botánicos de la siguiente página http://www.conabio.gob.mx/malezasdemexico/2inicio/home-malezas-mexico.htm.

...hay que dar agüita para que no seque la leche, porque hace secar la leche cuando entra frio a la espalda, por ahí conversando me dijeron que es bueno lecherón fregaron en la espalda y el agua de Paraguay. Persona del grupo indirecto que asesoro el uso de plantas a la mujer postparto 12 .

\section{DISCUSIÓN}

$\mathrm{A}$ nivel nacional e internacional es común el uso de plantas medicinales durante le postparto $(7,10)$. En la mayoría de naciones en donde existen pueblos y grupos aborígenes hasta la actualidad se utiliza medicina herbaria para mejorar y preservar la salud (11). El uso de plantas medicinales en este caso está relacionado con la creencia y la economía de los pueblos. Los países Asiáticos como Malasia y Africanos como Nigeria $(10,12)$ son los que en mayor cantidad registran investigaciones del uso de plantas. Países desarrollados y altamente tecnológicos el uso de plantas medicinales tiene una visión conservadora. Se encuentra relacionado el uso para evitar el daño que puede ocasionar los fármacos durante la lactancia materna (7).

El uso de infusiones con efecto uterotónicos que promueven la eliminación de loquios es evidenciado en otros estudios. El Tacca chantrieri André es una mezcla de Zingiber, officinale Roscoe y Alpinia galanga utilizada para estos fines (8). En algunos países de Asia utilizan Pimpenella anisum, Curcuma longa, Zingiber officinale entre las especies más destacadas (13). En Sudamérica es frecuente el uso de canela, anís, menta y salvia (14). En el Ecuador se destaca el uso de la paja blanca, calahuala, matico y nogal para la elaboración de la Purga. Sin embargo, entre las diferentes especies y preparados a nivel mundial se destaca el uso de plantas pertenecientes a la familia 
de las Asteraceae como uterotónicos $(7,13,15,16)$.

El uso de plantas medicinales para fines desinflamantes por infusiones para beber es común en otros países (17). El uso en infusiones tópicas para medio de aseo diario está muy poco documentado al respecto pero en países de América latina esta difundido la limpieza de heridas con infusión de manzanilla y matico (14). Estas plantas son desinflamantes y ayudan en la prevención de infecciones y la renovación (18). En Filipinas utilizan diferentes tipos de emplastos y tinturas para ser aplicadas en zonas genital y heridas como forma de sanación (19).

Las plantas medicinales para el baño es propio de países del Altiplano como Perú y Bolivia (20). La creencia establece que en zonas frías hay riesgo de resfriados, por este motivo el baño es un ritual cálido para mejorar el cuerpo (21). En países de zonas cálidas el año es de forma temprana para aseo corporal (22). Sin embargo, en la mayor parte del mundo el baño es de carácter relajante. Donde la utilización de esencias especiales fomentan la recuperación y relajación (10).

El uso de plantas como secretagogo tal vez sea la forma más utilizada y difundida a nivel mundial (11). Entre las complicaciones más identificadas es la hipogalactia por las implicaciones maternas sociales y alimentarias del recién nacido (23). Diversos estudios mencionan como principales galactagogos orales naturales (flor de plátano, hinojo, fenogreco, jengibre, ixbut, algodón herbáceo, moringa, dátiles de palma, codillo de cerdo, shatavari, silimarina, hojas de torbangun $\mathrm{y}$ otras mezclas naturales) (24). Un estudio estandarizado doble ciego controlado con placebo demostró eficacia en el uso de pastillas de jengibre de $500 \mathrm{mg}$ para aumentar los niveles de prolactina en el cuerpo (25).

\section{CONCLUSIONES}

$\mathrm{L}$ a investigación demuestra que la salud de la mujer durante el postparto tiene un efecto directo sobre la situación social y cultural de las familias. El uso de plantas medicinales se encuentra difundido en la práctica diaria del cuidado de la dieta. El uso y la forma de preparación se establecen con experiencias y se trasmiten de generación en generación. El principal grupo de plantas utilizadas son las denominadas cálidas para recobrar la energía vital que se pierde durante el parto desde el punto de vista ancestral. Desde el punto de vista botánico es la familia de las Asteraceae que se emplea. El uso de las diferentes especies depende de la costumbre familiar, la disponibilidad de recursos económicos y el lugar donde se desarrolle por la variabilidad de flora que existe. Lasplantas son utilizadas en infusión para tomar y para lavar. Se destaca el uso de la purga como úterotonico, té secretagogo e infusiones para la limpieza general en el baño o limpieza local de partes íntimas y heridas. El personal de salud debe tomar en consideraciones estar particularidades de la comunidad, para brindar orientación en la práctica ancestral según su origen étnico y formación educativa.

- Conflicto de Interés. Los investigadores declaran que no tienen conflicto de interés y que la investigación se ejecutó bajo los más estrictos principios éticos. 


\section{REFERENCIAS BIBLIOGRÁFICAS}

1. Salud OM de. Estrategia de la Medicina Tradicional de la OMS. Ginebra; 2014

2. Yemele MD, Telefo PB, Lienou LL, Tagne SR, Fodouop CSP, Goka CS, et al. Ethnobotanical survey of medicinal plants used for pregnant womens health conditions in Menoua divisionWest Cameroon. J Ethnopharmacol. 2015 Feb 3;160:14-31

3. Barnes LAJ, Barclay L, McCaffery $\mathrm{K}$, Aslani P. Complementary medicine products used in pregnancy and lactation and an examination of the information sources accessed pertaining to maternal health literacy: A systematic review of qualitative studies. BMC Complement Altern Med. 2018 Jul 31;18(1)

4. Zamawe C, King C, Jennings HM, Mandiwa C, Fottrell E. Effectiveness and safety of herbal medicines for induction of labour: A systematic review and meta-analysis. Vol. 8, BMJ Open. BMJ Publishing Group; 2018

5. Santoso EA, Jumari J, Utami S. Inventory of Medicinal Plants for Pregnant and Postpartum Women in Dayak Tomun of The Lopus Village Lamandau Regency of Central Kalimantan. Biosaintifika J Biol Biol Educ. 2019 Apr 1;11(1):25-31

6. Dewey K, Nommsen RL, Heining MJ CR. Evaluacion de la dieta y la nutricion de la Poblacion Urbana y Prevalencia determinate de la Hipertension y diabetis entre adultos en la India

7. Silalahi M, Khairiah A, Nisyawati. Ethnomedicinal plants and practices related to pregnancy, childbirth, and postpartum healthcare of minangkabau ethnic group, West Sumatra, Indonesia. Biodiversitas. 2020 Oct 1;21(10):4597-605

8. Bustamante G, Mantilla B, CabreraBarona P, Barragán E, Soria S, Quizhpe $\mathrm{E}$, et al. Awareness of obstetric warning signs in Ecuador: a crosssectional study. Public Health. 2019 Jul 1;172:52-60

9. Ministerio de Salud Pública del Ecuador (MSP). Mortalidad Evitable
Gaceta de Muerte Materna. Minist Salud [Internet]. 2018;1-10. Available from: https://www.salud.gob.ec/wpcontent/uploads/2017/07/GacetaSE-52-MM.pdf

10. Nik Yusof Fuad NF, Ching SM, Awg Dzulkarnain DH, Cheong AT, Zakaria ZA. Complementary alternative medicine use among postpartum mothers in a primary care setting: a cross-sectional study in Malaysia. BMC Complement Med Ther. 2020 Dec;20(1).

11. Özalkaya E, Aslandoğdu Z, Özkoral A, Topcuoğlu S, Karatekin G. Effect of a galactagogue herbal tea on breast milk production and prolactin secretion by mothers of preterm babies. Niger J Clin Pract. 2018 Jan 1;21(1):38-42

12. Nurrachmawati $A$, Wattie AM, Hakimi M, Utarini A. "Kamboh": A Qualitative Study Of Postpartum Care In Kutai Ethnic Tribe, East Kalimantan, Indonesia [Internet]. Vol. 19, Malaysian Journal of Public Health Medicine. 2019. Available from: http://mjphm.org/index.php/mjphm/ article/view/33

13. Ijioma SN, Osim EE, Nwankwo AA, Kanu KC, Orieke D. Southeast Nigerian polyherbal (AJUMBISE): A potential uterotonic and tocolytic agents. Sci African. 2020 Jul 1;8.

14. Lara-Cinisomo $S$, Wood J, Fujimoto EM. A systematic review of cultural orientation and perinatal depression in Latina women: are acculturation, Marianismo, and religiosity risks or protective factors? Vol. 22, Archives of Women's Mental Health. SpringerVerlag Wien; 2019. p. 557-67.

15 Karemore MN, Avari JG. Herbal Medicines Used During Pregnancy, Childbirth And Postpartum Care. Int J Pharm Sci Res [Internet]. 2017;8(12):5326-35. Available from: www.ijpsr.com

16. Kinyua EW, Maina CI, Kaluwa Kaingu C, Wafula Dk. Evaluation Of Oxytocin Like Effects Of Uvariodendron Kirkii (Verdec.) Extracts On Isolated Uterine Strips Of Wistar Rats. 2020; 
Available

from: http://dx.doi.org/10.5667/tang.2020. 0002

17. Khambaya $\mathrm{K}$, Zhang L, Kang $\mathrm{Y}$, Li H, Wang L, Muththanthirige $\mathrm{N}$, et al. Ethnobotanical Survey of Local Wisdom Knowledge on Medicinal Plants Used by the Traditional Phouthai Ethnic Group in NaKeu Village, Hinboun District, Khammouan Province, Lao PDR. Available from: https://doi.org/10.21203/rs.3.rs54642/v1

18. Conceição EPS, Kaezer AR, PeixotoSilva N, Felzenszwalb I, De Oliveira E, Moura EG, et al. Effects of Ilex paraguariensis (yerba mate) on the hypothalamic signalling of insulin and leptin and liver dysfunction in adult rats overfed during lactation. J Dev Orig Health Dis. 2017 Feb 1;8(1):12332

19. Dapar MLG, Alejandro GJD, Meve U, Liede-Schumann S. Quantitative ethnopharmacological documentation and molecular confirmation of medicinal plants used by the Manobo tribe of Agusan del Sur, Philippines. J Ethnobiol Ethnomed. 2020 Mar 5;16(1)

20. Bedoya-Ruiz LA, Agudelo-Suárez AA, Restrepo-Ochoa DA. Women in pregnancy, delivery, and postpartum: A look from the feminist point of view. Rev Peru Med Exp Salud Publica. 2020;37(1):142-7

21. Villavicencio EA, Rigoberto González J. Prácticas interculturales en el posparto; Tixán 2019 [Internet]. Universidad de Cueca; 2020. Available from:

https://dspace.ucuenca.edu.ec/bitstre
am/123456789/33795/1/PRACTICA

$S$ INTERCULTURALES EN EL PUERPERIO TIXAN 2019.docx.pdf

22. Fok D, Manager FA. A Comparison of Practices During the Confinement Period among Chinese, Malay, and Indian Mothers in Singapore. Eur PMC Funders Gr [Internet]. 2016;43(3):247-54. Available from: https://europepmc.org/article/pmc/ 4992357

23. Foong SC, Tan ML, Foong WC, Marasco LA, Ho JJ, Ong JH. Oral galactagogues (natural therapies or drugs) for increasing breast milk production in mothers of non-hospitalised term infants. Vol. 2020, Cochrane Database of Systematic Reviews. John Wiley and Sons Ltd; 2020

24. Foong $S C$, Tan ML, Foong $W C$, Marasco LA, Ho JJ, Ong JH. Oral galactagogues (natural therapies or drugs) for increasing breast milk production in mothers of non-hospitalised term infants. Cochrane Database Syst Rev. 2020 May 18;5(5):CD011505. doi: 10.1002/14651858.CD011505.pub2.

PMID: 32421208; PMCID:
PMC7388198

25. Paritakul $P$, Ruangrongmorakot $K$, Laosooksathit W, Suksamarnwong M, Puapornpong P. The Effect of Ginger on Breast Milk Volume in the Early Postpartum Period: A Randomized, Double-Blind Controlled Trial. Breastfeed Med. 2016 Sep;11:361-5. doi: $10.1089 / \mathrm{bfm} .2016 .0073$. Epub 2016 Aug 9. PMID: 27505611 